\title{
Re-audit of referral compliance of chlamydia-positive women from a family planning clinic
}

\begin{abstract}
Farzana Siddiqui, MRCOG, MFFP, Specialist Registrar in Sexual and Reproductive Health, The Palatine Centre, Manchester, UK; Rosemary J Kirkman, FRCOG, MFFP, Senior Lecturer in Family Planning, University of Manchester, Manchester, UK; Swatantrata Chandiok, MD, FRCOG, Consultant in Genitourinary Medicine, Withington Hospital, Manchester, UK
\end{abstract}

Correspondence: Dr Farzana Siddiqui, The Palatine Centre, 63-65 Palatine Road, Withington, Manchester M20 2XD, UK. E-mail: farzana.siddiqui@centralpct.manchester.nwest.nhs.uk

(Accepted 28 October 2003)

Journal of Family Planning and Reproductive Health Care 2004; 30(2): 86-87

\begin{abstract}
Background Chlamydia infections represent a major public health problem, with a prevalence of 6-10\% in family planning clinic (FPC) attendees. There has been recent concern expressed about the management of these patients in terms of treatment and follow-up.

Objective An audit was carried out to monitor referral compliance and outcome with care pathways of women attending our FPC who were found to be positive for chlamydia.

Setting The Palatine Centre FPC and genitourinary medicine (GUM) clinics in Manchester, UK.

Design Analysis of case notes was undertaken of women who tested positive for chlamydia between 1 January 2000 and 31 December 2000.

Results Of the 1935 women who were tested for chlamydia, $5.1 \%(n=99)$ were positive. The age range was $15-41$ (mean, 25) years. Treatment was verified in 90\% $(n=89)$ of cases, of which $85 \%(n=84)$ attended a GUM clinic. Despite reasonable efforts, information on outcome was not obtainable for 10 women. The median time between referral and treatment was 5-6 days. Sexual contacts were traced in $57 \%(n=56)$ of cases.

Conclusions We found our referral compliance and treatment rates to be excellent, demonstrating that GUM and family planning services can work well in partnership from different locations. However, this audit has shown low performance in partner notification and we recommend that more effort be directed to this aspect.
\end{abstract}

\section{Key message points}

- Family planning and genitourinary medicine clinics worked well in partnership even though not under 'one roof'.

- There is a need to examine ways to improve partner notification.

\section{Introduction}

In 1994, an audit conducted at The Palatine Centre family planning clinic (FPC) reported a prevalence of $4.35 \%$ chlamydia swabs positive on culture, with $89.3 \%$ of those women found positive actually attending the genitourinary medicine (GUM) clinic for treatment. ${ }^{1}$ Other studies have identified problems with the process of referral of patients who are found to be chlamydia-positive in a FPC with much lower rates of attendance at a GUM clinic. ${ }^{2-4}$ There is also a growing awareness of a need for closer liaison between departments of GUM and family planning services in order to promote sexual health.

The city of Manchester has three GUM clinics which are all part of acute hospital trusts, each having separate management and budgets. Some family planning doctors and nurses also work sessions at GUM clinics but there is at present no formal integration of the two services.

\section{Method and study population}

All women attending The Palatine Centre FPC, identified from the chlamydia swabs register to be positive during the 1-year period between 1 January 2000 and 31 December 2000, were included in this audit. A retrospective review of the women's case records was undertaken around the middle of 2001 in order to allow sufficient time for the women to attend for treatment and follow-up at the GUM clinic.

For the complete follow-up, health advisers based at the GUM clinics cross-referenced our list of referred family planning patients with the attendance records at the GUM clinics. The average waiting time to be seen at our nearest GUM clinic was 2 weeks whilst the others were reporting up to 4 weeks for routine appointments. ${ }^{5}$

\section{Results}

Of the 1935 swabs tested for chlamydia during the year, 99 $(5.1 \%)$ were positive. Documentation in the clinical records as well as in the swab register was found to be good. No results were reported missing.

The age range of women with confirmed chlamydia infection was 15-41 (median, 23) years. The majority of patients resided in Manchester $(\mathrm{n}=86,87 \%)$ with 13 women from outlying areas. The time period since the women first registered with the clinic ranged from that visit (19 women) to 16.8 years (median, 23 months).

Ninety-five percent $(n=94)$ of the patients had letters sent within 7 days of the test being taken. There was no permission given to contact the remaining five women, but all these individuals telephoned and received the result of the test. Ninety-two percent $(n=87)$ responded to the letter by either attending or phoning for the result. Seven of the non-responders definitely attended the GUM clinic for treatment. No information was obtainable for the remaining one woman who was resident out of the area. Therefore $99 \%(n=98)$ of the women definitely received their result.

Ninety percent $(n=89)$ of the women were known to have been treated for chlamydia. Eighty-five percent $(\mathrm{n}=$ $84)$ attended a GUM clinic, $4 \%(n=4)$ were treated by their

Table 1 Performance with respect to referral compliance and outcome as compared to standards recommended in the National Guidelines 6

\begin{tabular}{|c|c|c|c|}
\hline \multirow[t]{2}{*}{ Parameter } & \multicolumn{2}{|c|}{ Actual performance } & \multirow{2}{*}{$\begin{array}{l}\text { Performance } \\
\text { standard }\end{array}$} \\
\hline & 2001 & 1994 & \\
\hline Patients notified of results & $99 \%$ & $97 \%$ & $100 \%$ \\
\hline Patients treated & $90 \%$ & $89 \%$ & $100 \%$ \\
\hline Partner notification & 0.6 & $-\mathrm{a}$ & 0.7 \\
\hline
\end{tabular}

aNot measured 


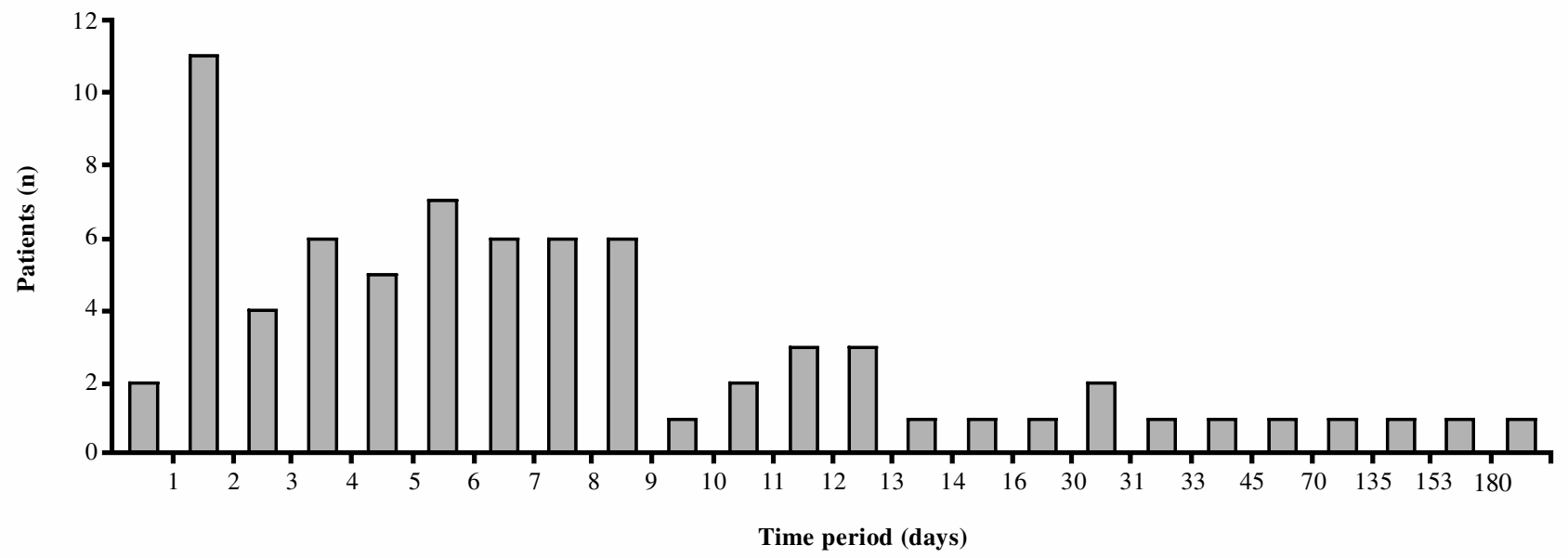

Figure 1 Time period between referral and attendance at a genitourinary medicine clinic

general practitioner (GP), one woman was treated in hospital prior to termination of pregnancy and no information could be obtained for the remaining 10 women.

There was a great variation in the length of time between referral by the FPC and attendance at the GUM clinic. The range was 1-180 days, with a median wait of 5-6 days (Figure 1).

Eighty-four women attended a GUM clinic, but only $56 / 84$ provided information on sexual partners, resulting in $57 \%(\mathrm{n}=56)$ named contacts who attended a GUM clinic for treatment and screening.

There was no information available on contact tracing for the four women who were treated by their GP.

\section{Discussion}

Ninety percent of the women were known to be treated for chlamydia, of which $85 \%(n=84)$ attended the GUM clinic. This represents a very good compliance with our referral procedures and also compares favourably with our previous audit. ${ }^{1}$

The majority of the women lived within 2 miles of both The Palatine Centre and the local GUM clinic at Withington Hospital. For $19 \%$ of women this was their first visit to the FPC. This could mean that the women who are having symptoms or are worried about a sexually transmitted infection prefer to attend the local FPC instead of their GP or the GUM clinic.

Our ratio of contacts seen per index case of 0.6 is low as compared to the 0.7 standard recommended in the National Guidelines. ${ }^{6}$ It was interesting that 28 women could not/would not contact or name their partner. We see this as the weakest point in our case management. In this context we are planning to undertake a pilot of initiating contact tracing slips from the FPCs and to monitor the outcome.

\section{Conclusions}

Referral compliance was found to be excellent, demonstrating that GUM and family planning services can work well in partnership from different geographical locations. However, this audit has shown low performance in partner notification and we recommend that more effort is directed to this aspect.

\section{Acknowledgements}

We would like to thank the health advisers at the Withington Hospital and Manchester Royal Infirmary GUM departments, and the clerical staff at The Palatine Centre for their invaluable contribution and help with the audit. We are indebted to Gretl McHugh, Research Fellow, for her comments and constant support.

Statements on funding and competing interests

Funding. None identified.

Competing interests. None identified.

References

1 Sin J, Gbolade BA, Russell A, et al. Referral compliance of chlamydia-positive patients from a family planning clinic. Br J Fam Plann 1996; 22: 155-156.

2 Tobin JM, Bateman J. Clinical audit of the process of referral to genitourinary medicine of patients found to be chlamydia-positive in a family planning service. Br J Fam Plann 1999; 24: 160-163.

3 Willmott $\mathrm{F}$, Tolcher $\mathrm{R}$. Audit of outcome following positive chlamydial test results in family planning clinics in Southampton. Int J STD AIDS 2000; 11: 756-758.

4 Wilkinson C, Massil H. An interface of chlamydia testing by community family planning clinics and referral to hospital genitourinary medicine clinics. Br J Fam Plann 2000; 26: 206-209.

5 Djuretic T, Catchpole M. Genitourinary medicine services in the UK are failing to meet current demand. Int J STD AIDS 2001; 12: 571-572.

6 FitzGerald MR, Welch J, Robinson AJ, et al. Clinical guidelines and standards for the management of uncomplicated genital chlamydial infection. Central Audit Group in Genitourinary Medicine. Int J STD AIDS 1998; 9: 253-262. 